\title{
Aspectos clínico-tomográficos da mucocele com invasão orbitária
}

\author{
Clinical and computed tomography aspects of mucocele with orbital invasion
}

$\begin{array}{lc}\text { Mary } & \text { Lúcia } \quad \text { Bedran } \\ \text { Jaime } & \text { Guedes }^{2} \\ \text { Vitor } & \text { Barbosa } \quad \text { Cerqueira } \\ \text { Viviane } & \text { Guedes } \\ \text { Edson } & \text { Marchiori }\end{array}$

Trabalho realizado no Hospital Universitário Clementino Fraga Filho da Universidade Federal do Rio de Janeiro (UFRJ) e no Hospital Universitário Antônio Pedro da Universidade Federal Fluminense (UFF).

${ }^{1}$ Professora Assistente do Departamento de Radiologia da Universidade Federal Fluminense - UFF. Mestre em Radiologia pela Universidade do Brasil - Universidade Federal do Rio de Janeiro - UFRJ.

${ }^{2}$ Pós-graduando do Serviço de Oftalmologia da Universidade do Brasil - Universidade Federal do Rio de Janeiro - UFRJ.

${ }^{3}$ Residente de Oftalmologia da Universidade do Brasil Universidade Federal do Rio de Janeiro - UFRJ.

${ }^{4}$ Ex-Fellow do Schepens Eye Research Institute, Harvard Medical School e do New England Medical Center, Tufts University School of Medicine Boston, MA, USA; Pós-graduanda em Oftalmologia, nível Doutorado, pela Universidade de São Paulo - USP; Médica do Instituto Benjamin Constant - RJ.

${ }_{5}^{5}$ Professor Titular do Departamento de Radiologia da Universidade Federal Fluminense-UFF; Coordenador do curso de Pós-graduação em Radiologia da Universidade do Brasil - Universidade Federal do Rio de Janeiro-UFRJ.

Endereço para correspondência: Dr. Jaime Guedes Av. das Américas, 500 Bl. 03 - Rio de Janeiro (RJ) CEP 22640-102 -E-mail: jaimeguedes@eyes.com.br

Recebido para publicação em 14.08.2001

Aceito para publicação em 07.04.2003

Nota Editorial: Pela análise deste trabalho e por sua anuência na divulgação desta nota, agradecemos ao Dr. Roberto Abucham.

\section{RES UMO}

Objetivo: Avaliar e relacionar os achados clínico-radiológicos da mucocele orbitária. Métodos: Análise clínica e de imagem referente a 166 pacientes com lesões expansivas da órbita examinadas com o tomógrafo Somaton DR da marca Siemens de terceira geração num período de 10 anos consecutivos em Hospital Universitário de referência. Resultados: Os achados clínicos mais comuns foram tumoração e proptose do globo ocular. À tomografia computadorizada, o achado típico foi de massa com baixa densidade, originando-se em seios paranasais, com destruição óssea adjacente, invasão da órbita e deslocamento do globo ocular. Conclusão: O desenvolvimento da tomografia computadorizada foi decisivo no estudo da órbita pois propicia, por meio de exame radiológico altamente especializado, imagens de incrível fidelidade anatômica revelando informações sobre o processo patológico como sua localização, sua relação com as estruturas adjacentes e sua vascularização.

Descritores: Mucocele/radiografia; Órbita/radiografia; Órbita/irrigação sangüínea; Tomografia computadorizada por raios x; Exoftalmia; Neoplasias orbitárias

INTRODUÇÃO

A órbita pode ser estudada por vários procedimentos geradores de imagem, como, por exemplo: radiografia convencional (RX simples), ultrasonografia, angiografia, doppler colorido, tomografia computadorizada (TC) e ressonância magnética $(\mathrm{RM})^{(1)}$ Destes, os dois principais são: TC e $\mathrm{RM}^{(1-2)}$.

A tomografia computadorizada teve nos anos de 1972/73 o início de sua aplicação clínica, causando uma verdadeira revolução no diagnóstico das patologias, em especial as orbitárias ${ }^{(3)}$. Pela grande diferença de densidade entre os músculos, nervos, vasos e a gordura retro-orbitária que envolve estes tecidos, a tomografia computadorizada mostrou-se um método extremamente valioso na avaliação da órbita ${ }^{(4)}$. É o método de escolha para avaliação de calcificações e das alterações ósseas da órbita. A tomografia computadorizada é melhor que a ressonância nuclear magnética para identificar corpos estranhos no trauma, definir textura, densidade e impregnação pelo meio de contraste ${ }^{(4-5)}$.

A ressonância nuclear magnética, por outro lado, é superior à tomografia computadorizada em outros aspectos, pois obtém imagens multiplanares que visibilizam melhor o ápice da órbita e a porção intracanalicular do nervo óptico, assim como oferece informações adicionais sobre a vascularização, além de caracterizar as hemorragias $^{(4)}$. Entretanto, possui certa limitação em relação à identificação de calcificações, além de ser um método de custo mais elevado.

A mucocele é a lesão expansiva mais freqüente dos seios paranasais. Ocorre com maior freqüência nos seios frontais, seguidos pelos etmoidais, maxilares e esfenoidais. É comum acometer mais de uma cavidade, envol- 
vendo os seios frontal e etmoidal, originando assim a mucocele fronto-etmoidal. Pode-se formar em cavidades de variação anatômica, como o processo pterigóide pneumatizado ${ }^{(6-7)}$. Sua incidência é em torno de 3,4\% entre os tumores orbitários. Pode surgir em pacientes de qualquer faixa etária, sendo mais freqüente em pacientes de meia idade ${ }^{(8)}$. Caracteriza-se por ser um tumor cístico, revestido internamente por uma membrana mucosa e repleto de muco, que se desenvolve a partir da obstrução do óstio do seio ${ }^{(6-9)}$. A etiologia pode ser infecciosa, traumática ou pós-cirúrgica ${ }^{(6)}$.

Classicamente, a mucocele apresenta sinais e sintomas resultantes do efeito de massa como, por exemplo, proptose do bulbo ocular e distúrbios visuais. Dor é uma condição rara e, quando presente, indica a presença de infecção (mucopiocele) ${ }^{(6-7,9)}$. O achado clínico mais comum é o de exoftalmia de progressão lenta ${ }^{(8)}$.

À tomografia computadorizada, apresenta-se em geral como lesão cística de conteúdo líquido, de baixa densidade (10 a 18 u.H.) e homogênea. Sua expansão gera remodelamento do tecido ósseo adjacente, com afinamento ósseo e perda deste tecido por provável isquemia secundária à compressão crônica do periósteo. Desta forma, a mucocele "invade" a cavidade orbitária, causando proptose e deslocamento das estruturas adjacentes ${ }^{(6-7,10)}$ (Fig. 1). Em alguns casos também se observa esclerose óssea. É importante lembrar que a mucocele não causa destruição óssea grosseira, o que ajuda a diferenciá-la dos carcinomas do seio da face e das lesões metastáticas, importantes diagnósticos diferenciais nas lesões das cavidades paranasais.

O objetivo deste trabalho é descrever os achados tomográficos compatíveis com mucocele orbitária observados em um período de 10 anos, num serviço de radiologia de um Hospital Universitário de referência.

\section{MÉTODOS}

Foi feito o levantamento dos exames tomográficos de órbita realizados no Serviço de Radiologia do Hospital Universitário Clementino Fraga Filho da Universidade do Brasil - UFRJ, no período de fevereiro de 1986 a fevereiro de 1995. Além disso, foram revistos os prontuários clínicos dos referidos pacientes que apresentavam lesão expansível da órbita à tomografia.

Os exames foram realizados com o tomógrafo modelo Somaton DR da marca Siemens, terceira geração.

A técnica preconizada para o estudo da órbita utiliza cortes axiais de 2-3 mm de espessura paralelos à linha de Reid (10 graus podálicos em relação à linha órbito-meatal), considerado o melhor ângulo para visualizar o nervo óptico intra-orbital e ver o conteúdo da órbita. Os cortes coronais usualmente complementam os cortes axiais, sendo realizados em posição prona com a cabeça pendendo para trás, de modo que esta fique estendida 70 graus. Devido à grande diferença de densidade que existe naturalmente entre os conteúdos orbitários, o contraste endovenoso não é necessário. Exceção se faz nos casos em que este pode ajudar na caracterização das lesões, como por exemplo, as vasculares, neoplásicas ou inflamatórias com suspeita de envolvimento intracraniano e também quando a espessura do nervo óptico precisa ser definida ${ }^{(2)}$.

\section{RESULTADOS}

No período de fevereiro de 1986 a fevereiro de 1995 foram realizados no Serviço de Radiologia do HUCFF -UFRJ um total de 360 exames de órbita em 344 pacientes (16 exames foram feitos como acompanhamento de 11 pacientes). Deste total, 166 pacientes $(48,25 \%)$ apresentaram lesões expansivas da órbita. Foram excluídos 20 pacientes dos quais não foram conseguidos nem o prontuário nem o exame tomográfico.

Dos 166 pacientes com lesões expansivas da órbita, oito apresentaram mucocele $(4,82 \%)$, sendo o diagnóstico confirmado por exame anátomo-patológico em seis casos $(75 \%)$ e por observação clínico-cirúrgica em outros dois (25\%). Quatro eram do sexo feminino e quatro do masculino (1:1), com idade variando de 24 a 69 anos, numa média de 48 anos. O quadro
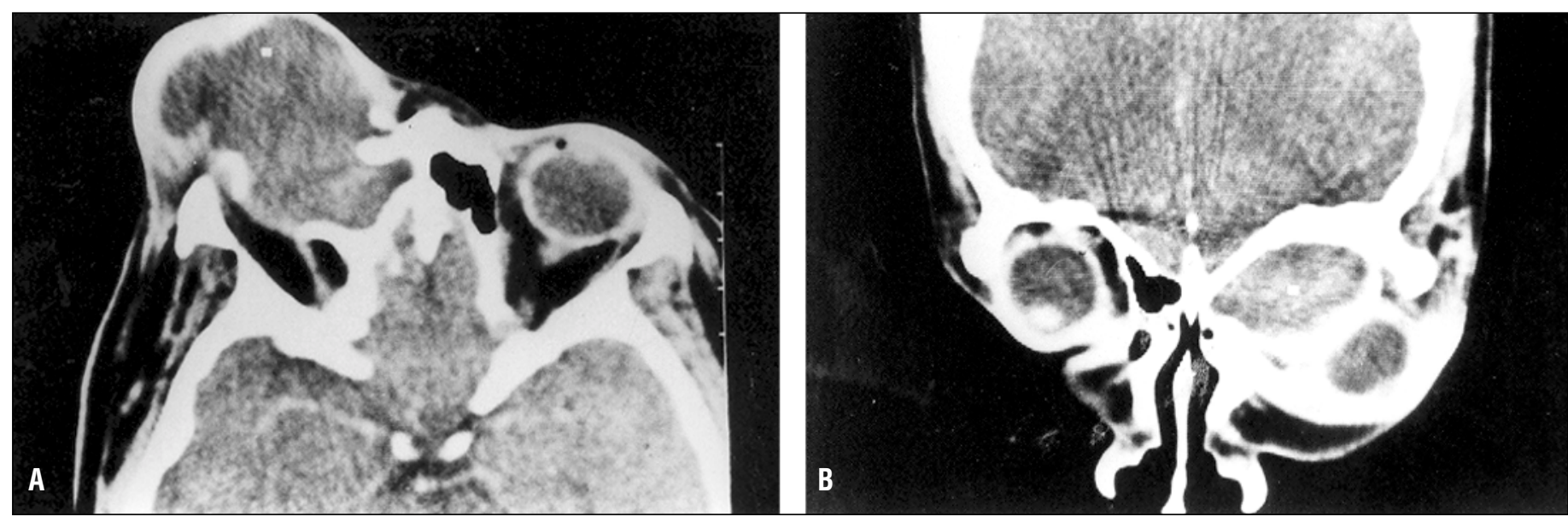

Fig. 1 - A: corte axial com contraste evidenciando massa hipodensa com impregnação periférica do meio de contraste, originando-se no seio frontal esquerdo com extensão exofítica para a órbita e destruição óssea adjacente; B: corte coronal com contraste onde a massa desloca o globo ocular inferior e lateralmente. Mucocele frontal esquerda 
clínico apresentado foi de proptose do globo ocular (50\%), tumoração (50\%), diminuição da acuidade visual (12,5\%), queda palpebral $(12,5 \%)$, cefaléia $(12,5 \%)$, perda da visão $(12,5 \%)$ e diplopia $(12,5 \%)$. Havia três pacientes com mucocele de origem frontal $(37,5 \%)$ e três com origem etmoidal $(37,5 \%)$, e duas fronto-etmoidais (25\%). A tomografia revelava massa com destruição óssea adjacente e desvio do globo ocular, em todos os casos. Houve impregnação periférica do meio de contraste em dois pacientes $(25 \%)$, já que havia mucosa inflamada.

Em relação aos tumores, de maneira geral, a mucocele ocupou o quinto lugar $(4,82 \%)$ e, em relação aos cistos, ocupou o primeiro lugar ( $80 \%$ das lesões císticas).

\section{DISCUSSÃO}

Dos oito pacientes, quatro eram do sexo feminino e quatro do masculino (1:1), com idades entre 24 a 69 anos, média de 48 anos.

Segundo Gonçalves et al ${ }^{(8)}$, as mucoceles correspondem a $3,4 \%$ das lesões expansivas orbitárias. A mucocele ocorre com mais freqüência em pacientes de meia idade.

Havia três pacientes com mucocele de origem frontal $(37,5 \%)$ e três com origem etmoidal $(37,5 \%)$, e dois onde o acometimento ocorria em duas cavidades frontal e etmoidal (25\%). Estes achados são compatíveis com os da literatura, que aponta maior ocorrência da mucocele nos seios frontais, em seguida nos etmoidais, acometimento simultâneo dos seios frontal e etmoidal, maxilares e esfenoidais ${ }^{(6,10-14)}$

Os achados clínicos mais comumente encontrados foram proptose do globo ocular (50\%) e tumoração (50\%), seguidos de diminuição da acuidade visual, queda palpebral, cefaléia, perda de visão e diplopia, cada um com $12,5 \%$.

O quadro clínico descrito na literatura também não difere do que foi encontrado neste trabalho. Classicamente, há sinais e sintomas resultantes de uma massa, com proptose do globo ocular e distúrbios visuais. O achado clínico mais comum é a exoftalmia, de progressão lenta que tipicamente causa proptose e deslocamento lateral do globo ocular ${ }^{(6-9)}$ (Fig. 2). Se houver elevação do assoalho orbital na mucocele maxilar, ocorre diplopia $^{(7)}$. As mucoceles esfenoidais são raras, e devido à possibilidade de acometer estruturas adjacentes (tais como os pares cranianos, seios cavernosos, artéria carótida interna, hipófise e quiasma óptico) suas manifestações clínicas são muito variadas ${ }^{(10)}$.

À tomografia computadorizada, encontramos massa com destruição óssea adjacente e desvio do globo ocular, dependendo da localização da mucocele (Fig. 3). É importante lembrar que a mucocele não causa destruição óssea grosseira, o que ajuda a diferenciá-la dos carcinomas dos seios da face e de lesões metastáticas. Outros achados descritos na literatura são o remodelamento ósseo (com afinamento e perda de tecido por isquemia secundária à compressão crônica do periósteo) e a esclerose óssea, que puderam ser observados neste estudo.

Houve impregnação pelo meio de contraste em dois pacientes (25\%), decorrente da presença de mucosa inflamada.

A literatura também é compatível com nossos achados. A mucocele usualmente se manifesta como massa expansiva de conteúdo líquido de baixa densidade (10 a 18 u. H.), homogênea; porém, em poucos casos, seu conteúdo pode ser mais viscoso e/ou conter mais proteína, elevando assim sua densidade para 20 a 40 u. H., e que, dependendo de sua localização, desvia o globo ocular ${ }^{(6-9)}$. Quando acomete o seio frontal, por exemplo, ocorre proptose ocular com deslocamento deste para baixo. A mucocele etmoidal é predominantemente anterior, talvez devido ao fato deste óstio ser o menor de todos. A viscosidade do muco também atua na gênese do processo, o que pode explicar porque a mucocele etmoidal é a mais comum em pacientes com mucoviscidose ${ }^{(11-14)}$. As mucoceles podem ser múltiplas quando decorrentes de traumatismo facial.

De regra, não ocorre impregnação do meio de contraste à tomografia computadorizada, nem do agente paramagnético à ressonância nuclear magnética. Quando isto ocorre, significa a presença de mucosa inflamada ${ }^{(6-7,10)}$.
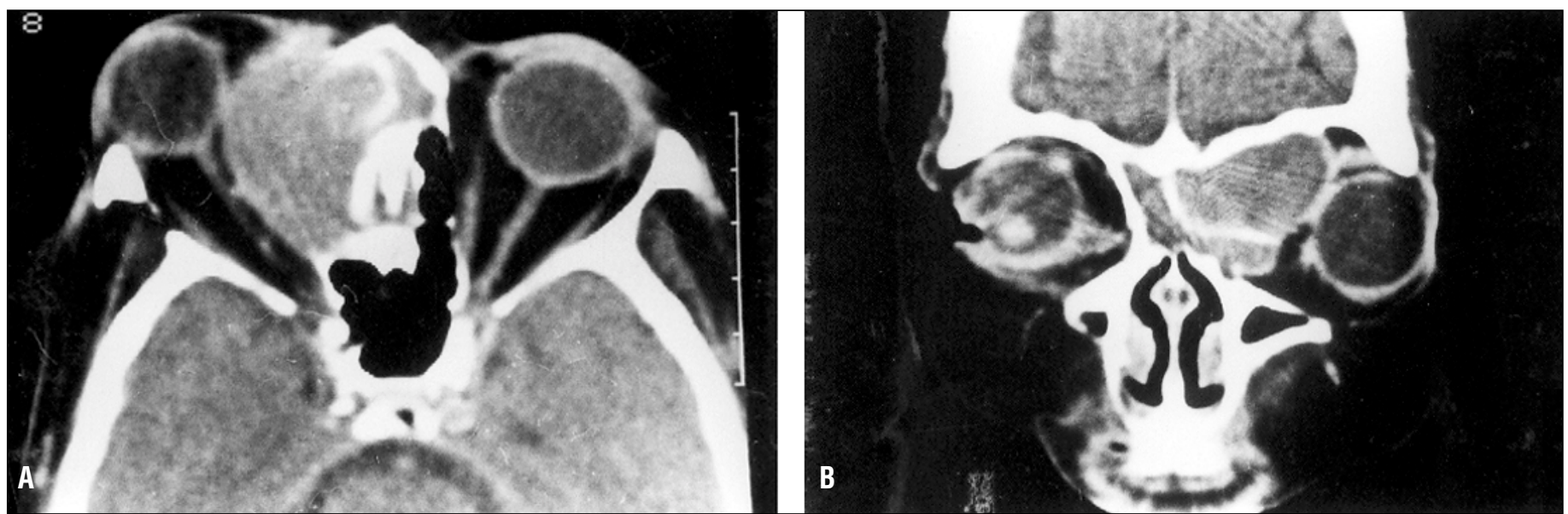

Fig. 2 - A: corte axial sem contraste evidenciando lesão expansiva hipodensa crescendo a partir das células etmoidais à esquerda com destruição das estruturas ósseas adjacentes, determinando desvio lateral e anterior do globo ocular; B: corte coronal após administração do meio de contraste venoso demonstra a massa desviando o globo ocular lateralmente. Mucocele fronto-etmoidal esquerda 

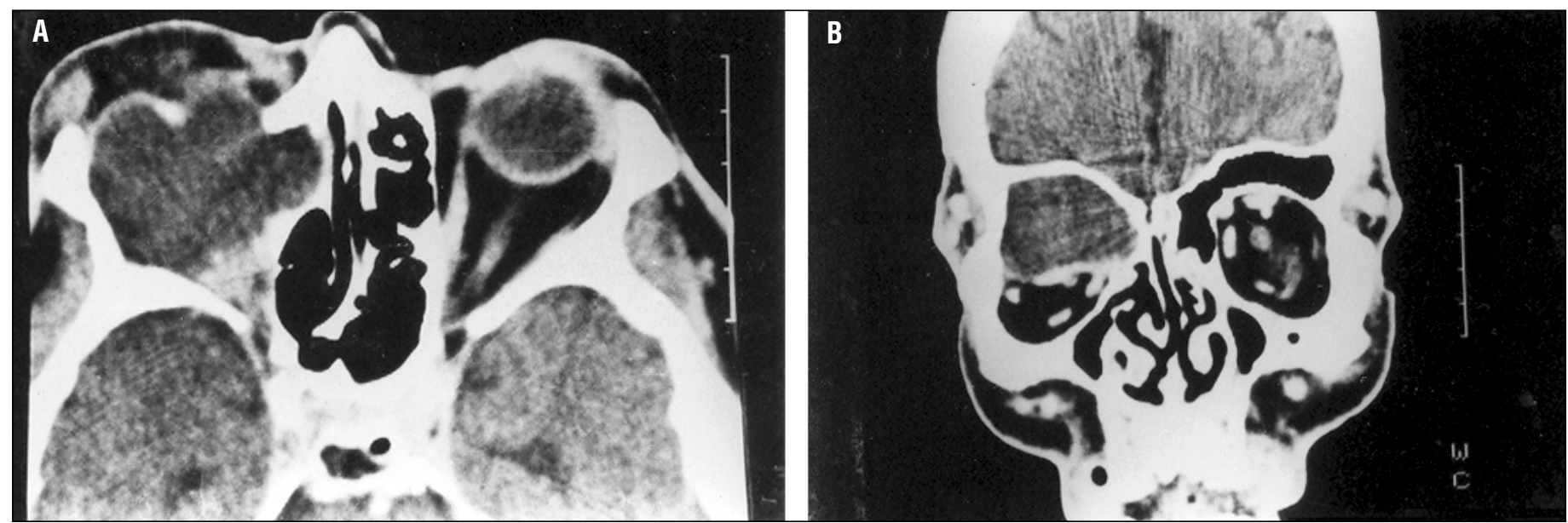

Fig. 3 - A: corte axial com contraste venoso evidenciando lesão expansiva no seio frontal esquerdo estendendo-se às células etmoidais deste lado, havendo destruição parcial da parede medial orbitária homolateral; B: corte coronal com contraste onde se observa que a massa rebaixa o teto da órbita esquerda. A referida massa se impregna perifericamente pelo meio de contraste. Mucocele fronto-etmoidal esquerda

O diagnóstico diferencial se faz, dentre outras lesões malignas, com schwannomas e linfomas das cavidades paranasais; estes, contudo, sofrem impregnação intensa e homogênea pelo meio de contraste ${ }^{(8-9)}$.

\section{CONCLUSÕES}

A tomografia computadorizada, que vem sendo utilizada clinicamente há três décadas, é de grande importância para o estudo das patologias orbitárias. Ela é capaz de fornecer imagens com extrema fidelidade anatômica.

Neste estudo descritivo retrospectivo de 166 casos, os resultados obtidos equivalem aos resultados da literatura.

É importante o diagnóstico preciso de mucocele orbitária uma vez que a conduta difere daquela adotada frente às principais patologias que fazem parte do seu diagnóstico diferencial, como os carcinomas do seio da face e as metástases, podendo definir a sobrevida ou não do paciente.

A tomografia computadorizada é portanto um método de imagem importante no diagnóstico das afecções dos seios da face, permitindo diferenciar as mucoceles de outras afecções benignas das cavidades paranasais como o papiloma invertido e os osteomas, bem como de lesões malignas primárias como os carcinomas e de outras tumorações metastáticas.

\section{A B S T RAC T}

Purpose: To analyze and establish clinical-radiological findings of orbital mucocele. Methods: Clinical analysis and image of 166 patients with expansive orbital lesions submitted to third generation Siemens Somaton DR computed tomography from February 1986 to February 1995. Results: The most common clinical findings were mass and proptosis. On computed tomography, the typical finding was mass with low density, coming from paranasal sinuses presenting adjacent bone des- truction and orbital invasion, with displacement of ocular globe. Conclusion: The development of computed tomography was an important mark in orbital studies because it allows incredible anatomic imagings, yielding information about the pathologic process like its localization and anatomic relation.

Keywords: Mucocele/radiography; Orbit/radiography; Orbit/ blood supply; Tomography, x-ray computed; Exophthalmos; Orbital neoplasms

\section{REFERÊNCIAS}

1. Santos AC, Prado PTC, Velasco e Cruz AA. Órbita: II - imagem. Arq Bras Oftalmol 1999;62:208-19.

2. Bedran ML, Guedes J, Cerqueira VB, Marchiori E, Guedes V. Aspectos tomográficos na celulite orbitária. Rev Bras Oftalmol 2000;59:631-5.

3. Manso PG. Tomografia computadorizada e ressonância nuclear magnética. Arq Bras Oftalmol 1995;58:495-8.

4. Rothfus WE. Normal orbit anatomy and CT scanning techniques. In: Latchaw RE, editor. Computed tomography of the head, neck and spine. Chicago: Year Book Medical; 1985. p. 361-6.

5. Bedran ML, Machiori E. Linfoma orbital: aspectos na tomografia computadorizada. Rev Bras Oftalmol 1998;57:55-8.

6. Florencio FT, Domenicio Jr O, Mendonça RA, Amaral LLF, Haetinger RG, Lima SS. Mucoceles: aspecto de diagnóstico por imagem. Estudo de 47 casos. Rev Imagem 1994;16:81-7.

7. Lloyd GAS. Diagnostic imaging of the nose and paranasal sinuses. J Laryngol Otol 1989;103:453-60.

8. Gonçalves JOR, Rocha H, Silva FA, editores Patologia da órbita. Rio de Janeiro: Livro Médico; 1984 (Biblioteca do Instituto Hilton Rocha, 1).

9. Perugini S, Pasquini U, Menichelli F. Mucoceles of paranasal sinuses envolving the orbit; CT signs in 43 cases. Neuroradiology 1982;34:133-9.

10. Dawson R III, Horton JA. MR. Imaging of mucoceles of the sphenoid sinus. AJR Am J Neuroradiol 1989;10:613-4.

11. Canalis RF, Jenkis HA. Ethmoid mucoceles. Arch Otolaryngol 1978;104: 286-90.

12. Friedman DP, Rao VM, Flanders AE. Lesions causing a mass in the medial canthus of the orbit. CT and MR features. AJR Am J Neuroradiol 1993;160: 1095-9.

13. Lloyd GA, Bartram CI, Stanley P. Ethmoid mucoceles. J Br Radiol 1974;47: 646-51.

14. Ritter FN. The paranasal sinuses: anatomy and surgical technique. St. Louis: Mosby; 1973. 